\title{
A short review on liquisolid technology in anti-fungal drugs
}

\author{
Shaveta Sharma, Harpreet Kaur, Jyoti Singh, Rahul Yadav \\ Department of Pharmacy, Chandigarh College of Pharmacy, Landran, India \\ Received: 11-11-2021 / Revised Accepted: 19-12-2021 / Published: 02-01-2022
}

\begin{abstract}
This article is based on the mode of action of antifungals and their mechanisms of action and approach for the treatment of fungal diseases like Aspergillosis, Candidemia, Fusariosis, and coccidioidomycosis. There are many different types of mechanisms contribute to the development of resistance to antifungals. The mechanisms include alteration in the drug target, sterol biosynthesis, the reduction in the intercellular concentration of target enzyme, and to make too many copies of the substance of the antifungal drug target.
\end{abstract}

Keywords: Aspergillosis, Candidemia, Fusariosis, and coccidioidomycosis

\section{INTRODUCTION}

These are the drugs that are generally used to treat deep or systemic fungal infections. Also known as an anti-mycotic medication. It is also used to prevent mycosis such as athlete's foot, ringworm, candidiasis (thrush), serious systemic infections such as cryptococcal meningitis, and others. These drugs are generally prescribed by physicians, but few are available over the counter (OTC). So, as I mentioned earlier these are the drugs are also known as antimycotic medication, is a pharmaceutical fungicide or fungistatic used to treat and prevent mycosis such as athlete's foot, ringworm, candidiasis (thrush), serious systemic infections such as cryptococcal meningitis, and others. These drugs are usually obtained by the doctor's prescription, but a few are available over the counter (OTC). These are associated with the use of broad-spectrum antibiotics, corticosteroids, anticancer/ immunosuppressant drugs, dentures, indwelling catheters and implants, and emergence of AIDS[1]. As a result of breakdown of host defence mechanisms, saprophytic fungi easily invade living tissue. There are many topical antifungals have been available since the antiseptic era. Two important antibiotics:amphotericin B-to deal with systemic mycosis, and griseofulvin-to supplement attack on dermatophytes were introduced around 1960.The antifungal properties of flucytosine was noted in 1970, but it could serve only as a companion drug to amphotericin. The discovery of the Imidazoles in the mid of 1970s and Triazoles in 1980s. There are some new compounds like terbinafine have been added lately[1].

\section{FUNGAL DISEASE}

There are millions of fungal species, but only a few hundred of them can make people sick. Yeast, molds, and mushrooms are all types of fungi. Rashes, asthma, meningitis, lung infections and bloodstream infections are different types of illness caused by fungi. There are some most common fungal diseases are fungal nail infections, vaginal candidiasis, Ringworm, and candida infections of

Address for Correspondence: Shaveta Sharma, Assistant Professor, M. Pharmacy (Pharmaceutics), Chandigarh College of Pharmacy, Landran, Awarded Best Pharmacy College in Punjab; E-mail: shaveta.ccp@cgc.edu.in

How to Cite this Article: Shaveta Sharma, Harpreet Kaur, Jyoti Singh, Rahul Yadav. A short review on liquisolid technology in anti-fungal drugs. World J Pharm Sci 2022; 10(01): 144-150;

https://doi.org/10.54037/WJPS.2022.100116

Copyright: 2022@ The Author(s). This is an open access article distributed under the terms of the Creative Commons AttributionNonCommercial-ShareAlike 4.0 International License (CC BY-NC-SA), which allows re-users to distribute, remix, adapt, and build upon the material in any medium or format for noncommercial purposes only, and only so long as attribution is given to the creator. If you remix, adapt, or build upon the material, you must license the modified material under identical terms. 
the mouth, throat, and esophagus. Fungal diseases that affect people who live in or travel to certain areas Blastomycosis It is caused due to the fungus Blastomyces, which lives in moist soil in parts of the United States and Canada. Coccidiomycosis Also known as Valley Fever. Caused by coccidioides, which lives in the southern United States and parts of Mexico and Central and South America[2].

\section{Histoplasmosis}

Caused by the fungus Histoplasma, which lives in the environment with large amounts of bird or bat droppings[2]. Aspergillosis: It is an infection which is caused by Aspergillus. It is a usually a mold (a type of fungus) that lives indoors and outdoors. Most people breathe in Aspergillus spores every day without getting sick. However, people with weakened immune systems or lung diseases are at a higher risk of developing health problems due to Aspergillus. The types of health problems which are caused by Aspergillus include allergic reactions, lung infections, and infections in other organs[3]. Candidiasis: It is a fungal infection caused by a yeast (a type of fungus) called Candida. Some Candida species can cause infections in people; the most common is Candida albicans. Candida normally lives on the skin and inside the body, in places such as the mouth, throat, intestines, and vagina, without causing any problems. Candida can cause infections if it grows uncontrollably or if it penetrates deeply into the body (for example, into the bloodstream or into internal organs such as the kidney, heart or also brain)[4]. Invasive candidiasis: It is an infection caused by a yeast (a type of fungus) called Candida. Unlike Candida infections in the mouth and throat (also called "fungal infections") or vaginal "fungal infections", an invasive yeast infection is a serious infection that can affect the blood, heart, brain, eyes, bones, and so on other body parts[5]. Candidemia, is a Candida bloodstream infection, which is a common infection generally seen in the hospitalized patients.

\section{Most Common Risk Factors for developing} Fungal Infections

1. Living in hot and humid climates, sweating too much, or wearing damp clothes can result in the development of fungal infections.

2. A weak or compromised immunity due to underlying diseases like diabetes, HIV, cancer etc. which may can cause fungal infections.

3. Living in an unclean environment and not maintaining personal hygiene can cause fungal infections.
4. Wearing dirty clothes like unclean or unwashed socks and innerwear can lead to fungal infections[6].

5. Wearing clothes that are too tight can cause sweating, providing the necessary moisture for the accelerated growth of fungi.

\section{HOME REMEDIES FOR FUNGAL INFECTION}

Eat Yoghurt and Probiotics The yoghurt and other probiotics have an enough amount of good bacteria that help stave off many fungal infections. They fight with the microbes that cause these infections. And also, fermented foods are another options for the excellent source of probiotics. If these are not helping, you could use probiotic supplements that have more concentrated dosages of good bacteria [7]. Wash with Soap and Water Firstly, clean the affected area with soap and water twice daily before applying any home remedies or any other medication[7]. This will helps to control the spread of infection. Use Tea Tree Oil The Tea tree oil is naturally obtained and have antifungal and antibacterial property. Mix it with any carrier oil like coconut oil or olive oil and dab over the infected area about three to four times a day[7]. Tea tree oil used in most cases because it is one of the most effective home remedies to treat fungal infections.

\section{Use Turmeric}

Turmeric is a potent antimicrobial and antiinflammatory spice and they have both the property as antimicrobial and anti-inflammatory[7]. It is used by mix with a little water and apply over the infected area. To get benefits in the internal body environment, mix with warm water, or have turmeric tea.

\section{Garlic}

Garlic have an antimicrobial property. One of the most potent antifungal and antimicrobial herbs. It is used in fungal infection by crush a couple of garlic with some olive oil and make a paste[7]. And then apply to the infected area for about thirty minutes.

\section{MECHANISMOFANTIFUNGALDRUGS}

So basically, the fungal cell membranes have a sterol, which is known as ergosterol, which works by replacing cholesterol found in mammalian cell membranes [8]. So, an antifungal medications are that which selectively eliminates fungal pathogens from a host with minimal toxicity to the host.

General mechanism of action for anti-fungal drugs includes:

a) Cell wall formation inhibition.

b) Disruption of cell membrane.

c) Inhibition cell division. 
Inhibition of fungal cell biosynthesis has not been successful and effective. Attempts have been made to interfere with fungal cell wall synthesis, using some of the chemical agents, which have demonstrated excellent anti-fungal activity in vitro. However, failure to develop these agents to useful drugs has verified to be a difficult task and hence ultimately development of new agents to target $\beta$ -

Table I. Classification of Anti-Fungal Drugs glucan synthesis has been focused[8]. Echinocandins are the recent class of antifungal agents that act by inhibiting $\beta-(1-3) D$-glucan at the fungal cell wall level, resulting in complete disruption of the cell wall and consequently cell death. Depictsmechanism of action of some most popular antifungal drugs.

\begin{tabular}{|l|r|}
\hline Antibiotics & $\begin{array}{l}\text { 1. } \\
\text { Polyenes: Amphotericin B(AMB), Nystatin, Hamycin, } \\
\text { Natamycin (Pimaricin) } \\
\text { Heterocyclic benzofuran: Griseofulvin }\end{array}$ \\
\hline Antimetabolites & Flucytosine (5-FC) \\
\hline Azoles & $\begin{array}{l}\text { Imidazoles } \\
\text { i) } \quad \begin{array}{l}\text { (Topical): Clotrimazole, Econazole, Miconazole, } \\
\text { Oxiconazle } \\
\text { ii) Systemic: Ketoconazole } \\
\text { Triazoles: Systemic: fluconazole, Itraconazole, Voriconazole }\end{array}\end{array}$ \\
\hline Allylamine & Terbinafine \\
\hline Other Topical agents & $\begin{array}{l}\text { Tolnaflate, Undecylenic acid, Benzioc acid,Quiniodochlor, Ciclopirox } \\
\text { olamine, Butenafine, } \\
\text { Sod. Thiosulfate. }\end{array}$ \\
\hline
\end{tabular}

Table II: Liquisolid formulation of anti-fungal Drugs

\begin{tabular}{|l|l|}
\hline Imidazole & Triazole \\
\hline Ketoconazole & Itraconazole \\
\hline
\end{tabular}

Table III. Excipients used in Antifungal Liquisolid Formulations

\begin{tabular}{|l|l|l|l|l|}
\hline Drug & $\begin{array}{l}\text { Non- } \\
\text { volatile } \\
\text { solvent }\end{array}$ & Carrier & Coating & References \\
\hline Itraconazole & PEG 600 & Alfacel PH 200 & Aerosil 200 & {$[17]$} \\
\hline Ketoconazole & PEG 400 & $\begin{array}{l}\text { Microcrystalline } \\
\text { cellulose }\end{array}$ & $\begin{array}{l}\text { Colloidal } \\
\text { Silica }\end{array}$ & {$[18]$} \\
\hline
\end{tabular}

\section{MODE OF ACTION BY THEIR CLASSIFICATION \\ Polyene antibiotics}

The polyenes consist of a macrocyclic ring with many conjugated double bonds on one side and are highly lipophilic, while the other side is hydrophilic with many $\mathrm{OH}$ groups. Some have a polar amino sugar and a carboxylic acid group at one end.

A. Amphotericin B: Polyenes have a macrocyclic ring, one side of which has several conjugated double bonds and is highly lipophilic, while the other side is hydrophilic with many $\mathrm{OH}$ groups. A polar amino sugar and a carboxylic acid group are present at one end of some. All are soluble in water and unstable in an aqueous medium. The hydrophilic side forms the interior of the pore through which ions, amino acids and other water soluble substances move out. The cell permeability is markedly increased and due to this the cell death occurs.

B. Nystatin: Nystatin is derived from noursei and is similar to amphotericin B in antifungal and other properties. Binds to the fungal cell membrane (Ergosterol) and forms pores. This impairs permeability and transport and as a result cell death occurs.

Griseofulvin: It is extracted from the Penicillium griseofulvin. It interferes with the multinucleated mitosis and results to anaesthetized fungal hyphae which is done by its action. Then causes an abnormal metaphase configuration without causing metaphase arrest. It is mostly active against dermatophytes. 


\section{Antimetabolite}

Flucytosine: It is an inactive and a pyrimidine antimetabolite. It is taken up by fungal cells via the enzyme cytosine permease. $5-F C$ is then converted by a series of steps to 5-fluorodeoxyuridine 5'monophosphate. This false nucleotide inhibits thymidylate synthase, thereby depriving the organism of thymidylic acid, an essential DNA component. The unnatural mononucleotide is further metabolized to a trinucleotide (5fluorodeoxyuridine 5'-triphosphate) and is incorporated into fungal RNA, where it disrupts nucleic acid and protein synthesis.

Echinocandins: Echinocandins interfere with the synthesis of the fungal cell wall. It is Glucan synthesis inhibitor[9].It works by inhibiting 1,3beta glucan synthase, an enzyme important in fungal cell wall synthesis and subsequently inhibit the synthesis of beta glucan in the fungal cell wall. Disruption of the fungal cell wall leads to cellular osmotic instability and cell death.

Terbinafine: It is synthetic allyl-amine and orally active. It is used for treating dermatophytes, especially onychomycosis. It interferes with ergosterol biosynthesis by inhibiting the fungal enzyme squalene epoxidase rather than interacting with the P450 system. Acting as a structural analogue of squalene, terbinafine causes the accumulation of this unsaturated hydrocarbon, and a decrease in ergosterol in the fungal cell membrane. The accumulation of toxic amounts of squalene result in the death of the fungal cell[10].

Azoles:The azole antifungal drugs act by inhibiting the synthesis of the sterol components of the fungal membrane. Azoles are predominantly fungistatic. They inhibit C-14 $\alpha$-demethylase (a cytochrome P450 [CYP450] enzyme), thereby blocking the demethylation of lanosterol to ergosterol, the principal sterol of fungal membranes. This inhibition disrupts membrane structure and function, which then inhibits fungal cell growth[11].Azoles are primarily divided into two groups: Imidazoles and triazoles. Clotrimazole, Econazole, Miconazole, Ketoconazole fall under Imidazoles group and Fluconazole, Itraconazole, Posaconazole and Voriconazole fall under triazoles group The Imidazoles and triazoles cause rapid defects in fungal membrane integrity due to reduced levels of ergosterol, with loss of cytoplasmic constituents leading to similar effects to the polyenes[11].They impair ergosterol synthesis leading to cascade of membrane abnormalities in the fungus.

\section{USES}

Azole antifungal drugs in treatment of coccidioidomycosis

For two decades amphotericin B has been the sole antifungal drug with proven efficacy against Coccidioides immitis. The introduction of miconazole within the late 1970s ushered in a very new era of antifungal therapy ${ }^{[\mathbf{1 2}]}$. Miconazole induced remissions in patients with disease refractory to amphotericin B and in patients who had relapsed after amphotericin B treatment. Almost as important, miconazole failed to have the nephrotoxicity related to amphotericin B. However, the requirement of intravenous administration and other toxicities limited the worth of miconazole. The primary child of miconazole was ketoconazole, which may be administered orally and had markedly less toxicity than amphotericin B or miconazole. Because coccidioidomycosis challenges any antifungal drug, doses were increased to the maximum amount as $2,000 \mathrm{mg} / \mathrm{d}$. Ketoconazole are often used for long terms and at high doses, with many patients brought into remission. A $3^{\text {rd }}$ generation of azoles, the Triazoles, was introduced to attenuate these adverse effects, yet retain the efficacy of ketoconazole. Other triazoles with advantageous pharmacokinetic properties also are coming into clinical trials[12]. Shortly, we are likely to possess available full range of antifungal agents for treatment of this most frustrating disease.

\section{Treatment of documented invasive fungal disease \\ Candidemia}

There is little data on the treatment of candidemia in neutropenic patients. Among 10 randomized trials of various antifungal agents for the treatment of candidemia/invasive candidiasis, only five included neutropenic patients, and also the proportion of such patients was usually $<10 \%$. Taking these limitations into consideration, an echinocandin is taken into the account the drug of choice as primary treatment for Candidemia (Caspofungin $70 \mathrm{mg}$ on day 1 and $50 \mathrm{mg}$ thereafter; micafungin $100 \mathrm{mg}$ daily or anidulafungin $200 \mathrm{mg}$ on day 1 and $100 \mathrm{mg}$ thereafter). Step-down therapy to fluconazole (400 $\mathrm{mg}$ once a day) after some days of intravenous echinocandin may be a good alternative[13]. On condition that the patient is improving and also the isolateisn't $C$. glabrata or $C$. krusei. An alternate to an echinocandin is L-AMB ( $3 \mathrm{mg} / \mathrm{kg}$ daily). For the treatment of chronic disseminated candidiasis, LAMB followed by oral fluconazole or Voriconazole for prolonged periods is that the treatment of choice. The utilization of corticosteroids may accelerate clinical improvement. 
Aspergillosis

Voriconazole is the primary choice of drug for the treatment of invasive aspergillosis. And, treatment usually is started with the i.v. preparation $(6 \mathrm{mg} / \mathrm{kg}$ twice a day on day 1 and $4 \mathrm{mg} / \mathrm{kg}$ thereafter), although a study suggested that starting therapy with oral Voriconazole is not associated with poorer outcomes. A recent studies also suggested that higher doses of oral Voriconazole (300 to 400 $\mathrm{mg}$ twice a day) are needed in order to achieve therapeutic serum levels of the drug[13].

A recent randomized study compared Voriconazole with the combination of Voriconazole and anidulafungin in the treatment of invasive aspergillosis. The 6-week survival was $80.7 \%$ in patients receiving combination therapy and $72.5 \%$ in patients receiving Voriconazole ( $\mathrm{p}$-value $=0.08$ ). A sub-group analysis of patients with baseline positive serum galactomannan showed a statistically significant survival advantage of the combination arm[13].

Fusariosis

The outcome of invasive fusariosis is very poor, with a $21 \%$ 90-day probability of survival in patients with hematologic diseases and only $13 \%$ in HSCT recipients. The drug of choice is a lipid formulation of AMB. We recently analyzed the outcome of 158 cases of fusariosis, and observed that the outcome has improved in the last decade. Multivariate analysis showed that receipt of $d-$ AMB was associated with poor outcome. By contrast, survival was improved with the use of Voriconazole

\section{CHARACTERISTICS OF SELECTED ANTIFUNGAL DRUGS MARKETED IN INDIA}

Nystatin

It is an antifungal medicine. It is used to treat cutaneous, muco-cutaneous, and gastrointestinal mycotic infections, particularly those caused by Candida species. It is not absorbed orally and has not been linked to drug induced liver injury. Mycostatin (Sarabhai Company) is mainly marketed in India. It is available in two different dosage formulation that is suspension and tablet form(also in vaginal tablet) in India.

\section{Background}

Nystatin is a polyene antifungal drug that has broad-spectrum fungicidal and fungistatic activity against a number of yeasts and fungi, most notably Candida species. It is one of the most effective antifungal agents synthesized by bacteria, in this case a strain of Streptomyces noursei, and is closely related to amphotericin B, differing only slightly in structure. Nystatin has a greater antifungal activity than amphotericin B - parenterally administered nystatin, however, is associated with significant toxicity and is not available in a formulation appropriate for systemic use. As it undergoes very little absorption following oral or topical administration, nystatin's efficacy is limited to the treatment/prevention of cutaneous, mucocutaneous, and gastrointestinal fungal infections[14].

\section{Indication}

Nystatin is available in oral formulations for the treatment and/or prevention of oral candidiasis (a.k.a. thrush), intestinal candidiasis, and anal candidiasis. It is indicated topically for the treatment of vulvovaginal candidiasis and other cutaneous candida infections. A combination product containing nystatin alongside neomycin, gramicidin D, and triamcinolone is indicated in the treatment of corticosteroid-responsive dermatoses caused by bacterial. It is also available in combination with metronidazole for the treatment of mixed infections due to Trichomonas vaginalis and Candida albicans. Nystatin is also sometimes used off-label for the prevention of invasive candidiasis in low birth weight neonates, though it is generally reserved as a second-line option after fluconazole [14].

Mechanism of Action

Nystatin could be a channel-forming ionophore, meaning it exerts its therapeutic effect via formation of a membrane-spanning pore within the fungalsemipermeable membrane. The formation of this pore leads to a change in membrane permeability that permits for leakage of intracellular contents and also the subsequent disruption of electrochemical gradients necessary for correct cell function. Selectivity for fungal cells over mammalian cells is because of nystatin's greater binding affinity for ergosterol, a key sterol found in fungi cell walls, as criticalits mammalian counterpart, cholesterol[14].

Toxicity

The oral $\mathrm{LD}_{50}$ in rats is $10 \mathrm{~g} / \mathrm{kg}$. There are no reports of significant toxic effects following overdosage of nystatin - doses in more than5 million units daily have resulted in nausea and gastrointestinal upset with no other associated effects.

Ketoconazole

It is a broad spectrum antifungal used to treat seborrheic dermatitis and fungal skin infections.[15]

Background

Ketoconazole is an imidazole antifungal agent used in the prevention and treatment of a variety of fungal infections. It works by preventing the 
synthesis of ergosterol, the fungal equivalent of cholesterol, thereby increasing membrane fluidity and preventing growth of the fungus. It was first approved in an oral formulation for systemic use by the FDA in 1981. At this time it was considered much due to its broad spectrum and good absorption over previous antifungals, miconazole and Clotrimazole. After that, it was discovered that ketoconazole produces frequent gastrointestinal side effects and doserelated hepatitis. These effects combined with waning efficacy led to its eventual replacement by

Triazoles agents, fluconazole, Itraconazole, Voriconazole, and posaconazole. Ketoconazole and Clotrimazole continue to be used in topical formulations[15].

Mechanism of Action

Ketoconazole interacts with 14- $\alpha$-sterol demethylase, a cytochrome P-450 enzyme necessary for the conversion of lanosterol to ergosterol. Which results in the inhibition of ergosterol synthesis and increased fungal cellular permeability due to reduced amounts of ergosterol present in the membrane of fungal cell. And, this metabolic inhibition also results in accumulation of $14 \alpha$-methyl-3, 6-diol, which is a toxic metabolite. The increase in membrane fluidity is also thought to produce destruction of membrane-bound enzyme systems as components become less closely packed.

Toxicity

Acute liver injury, which may include both hepatocellular and cholestatic injury, accompanied by anorexia, fatigue, nausea, and jaundice are the symptoms of overdose of the ketoconazole. So, in case of the overdose, gastric lavage with activated charcoal may be used if within one hour of ketoconazole ingestion otherwise provide supportive care. If the patient shows signs of adrenal insufficiency, administer $100 \mathrm{mg}$ hydrocortisone once together with saline and glucose infusion and monitor the patient closely. After that blood pressure, fluid and electrolyte balance should be monitored over the next few days.

\section{TERBINAFINE}

It comes under the class of an Allylamine antifungal used to treat dermatophyte infections of toenails and finger-nails as well as other fungal skin infections.[16]

Background

Terbinafine hydrochloride (Lamisil) is a synthetic Allylamine antifungal. Terbinafine is highly lipophilic in nature and ability to accumulate in the skin, nails, and fatty tissues. Like other allylamines in the market, terbinafine inhibits ergosterol synthesis by inhibiting the fungal squalene monooxygenase which is also called squalene epoxidase, an enzyme that is part of the fungal cell wall synthesis pathway. Terbinafine hydrochloride was granted by FDA approval on 30 December 1992.

Indication

Terbinafine hydrochloride is used to treat fungal skin and nail infections caused by Trichophyton species, Microsporum canis, Epidermophyton

floccosum, and Tinea species. It is also treats yeast infections of the skin caused by Candida species and Malassezia furfur.

Mechanism of action

Terbinafine acts by inhibiting the enzyme squalene monooxygenase (also called squalene epoxidase), preventing the conversion of squalene to 2, 3oxydosqualene. It is a step in the synthesis of ergosterol. This inhibition leads to decreased ergosterol, which would normally be incorporated into the cell wall, and accumulation of squalene.

\section{Toxicity:}

Terbinafine induced liver injury because it is identified shortly after its introduction into medical use. It is mainly associated with elevations in serum aminotransferases in less than $1 \%$ of patients and the elevations are generally asymptomatic and resolve without stopping therapy. The symptoms are expected to be nausea, vomiting, stomach pain, rash, frequent urination, and headache. Activated charcoal, symptomatic and supportive therapy is used to treat overdose of terbinafine.

\section{ACKNOWLEDGMENT}

Authors are thankful to the management of Chandigarh University and Chandigarh Group of College Landran for providing facilities to give access for literature survey to do this review work.

\section{REFERANCE}

1. http://www.pharmacy180.com/article/antifungal-drugs-1283/

2. https://www.cdc.gov/fungal/about-fungal-diseases.html

3. https://www.cdc.gov/fungal/diseases/aspergillosis/index.html

4. https://www.cdc.gov/fungal/diseases/candidiasis/thrush/index.html

5. https://www.cdc.gov/fungal/diseases/candidiasis/genital/index.html 
6. H. Rehman et al. Deltamethrin Increases Candida albicans Infection Susceptibility in Mice. Scand J Immunol. 2011; 73(5) : 459-464

7. https://pharmeasy.in/blog/try-these-simple-home-remedies-for-fungal-infections/

8. Tripathi K.D. Essentials of Medical Pharmacology. $3^{\text {rd }}$ ed Jaypee Brothers Medical Publishers. 1994.

9. Glöckner A et al. Treatment of invasive candidiasis with echinocandins, Mycoses. 2009; 53(6) : 476486

10. Lam P.L. et al., Miconazole and terbinafine induced reactive oxygen species accumulation and topical toxicity in human keratinocytes, Drug Chem Toxicol 2020;14:1-5

11. https://microbiologyinfo.com/mode-action-antifungal-drugs/

12. https://pubmed.ncbi.nlm.nih.gov/3317599/

13. https://www.ncbi.nlm.nih.gov/pmc/articles/PMC3486829/

14. https://go.drugbank.com/drugs/DB00646

15. https://go.drugbank.com/drugs/DB01026

16. https://go.drugbank.com/drugs/DB00857 